(C) 2017. This manuscript version is made available under the CC-BY-NC-ND 4.0 license http://creativecommons.org/licenses/by-nc-nd/4.0/

This accepted version is restricted to registered institution users only until 2 August 2018.

Final published version is available as: Butler K, Klaus K, Edwards L and Pennington K. Elevated cortisol awakening response associated with early life stress and impaired executive function in healthy adult males. Hormones and Behavior. 2017; 95: 13-21. doi.org/10.1016/j.yhbeh.2017.07.013.

\title{
Elevated Cortisol Awakening Response Associated with Early Life Stress and Impaired Executive Function in Healthy Adult Males
}

Kevin Butler ${ }^{\mathrm{a}}$, Kristel Klaus ${ }^{\mathrm{a}}$, Laura Edwards ${ }^{\mathrm{a}}$, Kyla Pennington ${ }^{\mathrm{a}}$

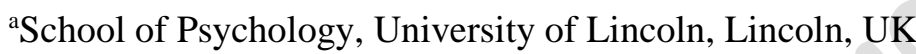

Address of corresponding author:

Dr Kyla Pennington

School of Psychology

Sarah Swift Building

University of Lincoln

Brayford Wharf East

Lincoln, LN5 7AY

UK

Email: kpennington@lincoln.ac.uk

Tel. +44 (0)1522 886199 


\begin{abstract}
Experiencing early life stress (ELS) and subsequent dysregulation of the hypothalamic-pituitaryadrenal (HPA) axis may play a role in the aetiology of mental health disorders. However, the exact mechanisms linking HPA-axis dysregulation with the development of psychopathology have not been fully delineated. Progress in this area is hampered by the complex and often conflicting associations found between markers of HPA-axis function and risk factors for mental health disorders such as impaired executive function (EF) and ELS. This study investigated the association of the cortisol awakening response (CAR) with ELS and EF in a healthy adult male population $(n=109$, aged 21-63). As previous inconsistencies in CAR and ELS association studies may be the result of not considering ELS-related factors such as cumulative exposure, type of stressor and developmental timing of ELS, these were also investigated. The main findings were that the CAR was significantly elevated in individuals reporting ELS compared to those reporting no ELS $(p=0.007)$ and that an elevated CAR predicted poorer problem solving/planning $(\mathrm{p}=0.046)$. Cumulative exposure, type of stressor and developmental timing of ELS were also found to impact significantly on the CAR. These results suggest that ELS is associated with chronic changes in HPA-axis function and that these changes may be associated with impairments in problem solving/planning. Future work should investigate further the neurobiological mechanisms linking ELS, the CAR and EF and their role in conferring risk for the development of mental health disorders.
\end{abstract}

Keywords: Cortisol awakening response; Early life stress; Executive function; Healthy adults; Planning; Problem solving. 


\section{Introduction}

Experiencing stressful life events during childhood or adolescence (early life stress; ELS) is a risk factor for later development of mental health disorders such as depression and schizophrenia (Mandelli et al., 2015; McLaughlin et al., 2010; Read et al., 2001). Although the exact mechanisms have not been fully delineated the hypothalamic-pituitary-adrenal (HPA) axis, the major neuroendocrine regulator of stress responses, likely plays a role in mediating the effect of stressors on the development of psychopathology (Doom and Gunnar, 2013). Impaired executive function (EF) may also be a risk factor for mental health disorders. Impairments in $\mathrm{EF}$, a set of higher order cognitive processes that facilitate the attainment of goals (Diamond, 2013), are transdiagnostically associated with mental health disorders (McTeague et al., 2016) that are linked with ELS. Indeed, impaired EF may be an intermediate phenotype more closely linked to biological mechanisms than more complex disease states per se (i.e. a cognitive endophenotype; Almasy and Blangero, 2001; Gottesman and Gould, 2003) and it may represent a mechanism linking a dysfunctional HPA-axis to mental health problems. Conversely, better EF may protect against the development of mental health disorder symptoms in 'at risk' populations (Davidovich et al., 2016). Consequently, studies that investigate both the effects of ELS on HPA-axis function during adulthood and the relationship between ELS-induced alterations of HPA-axis function and EF may be particularly important for understanding risk phenotypes that may confer vulnerability to psychopathology.

One common method of investigating HPA-axis function is through the measurement of the cortisol awakening response (CAR). Originally described in the 1990's (Pruessner et al., 1997) the CAR refers to a typical rise in cortisol within the first 30 minutes of waking (Clow et al., 2004; Wust et al., 2000). The CAR has been shown to be associated with mental health disorders, with an elevated CAR predicting subsequent new on-set anxiety (Adam et al., 2014) and depression (Adam et al., 2010), a blunted CAR being found in patients with severe depression symptomology (Veen et al., 2011) and those with first episode psychosis and schizophrenia (Berger et al., 2016). Since the CAR may be a stronger predictor of depression than other measures of HPA-axis activity (Adam et al., 2010) it may 
be a particularly interesting index of HPA-axis function for investigating risk factors for mental health disorders.

Studies investigating the physiological and cognitive effects of experiencing early life stress (ELS) in healthy adults enable investigation of potential risk phenotypes for psychopathology free from confounding factors associated with disease, such as symptoms and medication. Despite this, few studies have been conducted aiming to elucidate how the experience of ELS contributes to dysregulation of the CAR in non-psychiatric populations. Those that have show mixed findings regarding the association of ELS with CAR in adulthood, with both negative (Meinlschmidt and Heim, 2005) and positive (Engert et al., 2011) associations reported. It has been suggested that different types of stressor may have differential effects on HPA-axis function as assessed by the CAR (Engert et al., 2011). This is supported by work showing that physical abuse compared to emotional abuse may increase HPAaxis sensitivity to subsequent stressors (Kuhlman et al., 2015a). In addition, experiencing physical abuse is associated with a blunted cortisol response under acute stress challenge even when controlling for other types of ELS (Carpenter et al., 2011) and early physical/sexual abuse, but not other types of early onset maltreatment, has been linked with diurnal cortisol dysregulation (Cicchetti et al., 2010).

Several strands of research suggest that other ELS-related factors may lead to greater or lesser impacts on the functioning of the HPA axis. The allostatic load hypothesis predicts that chronic or repeated exposure to stress leads to a cumulative physiological toll that likely includes dysregulation of the neuroendocrine stress system (McEwen, 1998). Indeed previous research with children has found that those who had experienced multiple types of ELS were more likely to show hyperactivity of the HPA axis indexed by elevated morning cortisol levels (Cicchetti and Rogosch, 2001). The age that ELS is experienced may also be related to HPA-axis dysregulation. For example, early but not later onset of physical/sexual abuse is linked with diurnal cortisol dysregulation in children (Cicchetti et al., 2010). Furthermore, previous research has found that experiencing stressful life events during younger childhood as opposed to adolescence is associated with greater risk of developing depression or post- 
traumatic stress disorder (Maercker et al., 2004), and poorer response to antidepressant treatment in depressed patients (Williams et al., 2016).

Several studies have also focused on the effect of ELS exposure on executive function (EF). Studies typically report deficits in EF following ELS (e.g. Bos et al., 2009; Cardona et al., 2012; Loman et al., 2013). However childhood adversity may not lead to a general impairment in EF and could actually enhance specific aspects (Mittal et al., 2015). A dysregulated HPA-axis, as a consequence of ELS, is likely to affect EF since EFs are subserved by stress-sensitive brain regions that have a protracted development such as the prefrontal cortex (Pechtel and Pizzagalli, 2011). This protracted development alongside a high concentration of glucocorticoid receptors make this area vulnerable to both structural and functional changes induced by chronic stress (e.g. Arnsten, 2009; Brown et al., 2005; Izquierdo et al., 2006). However, findings from studies examining the relationship between the CAR and EF have been inconsistent with some studies showing positive associations (working memory: Almela et al., 2012; attention switching: Evans et al., 2012; Law et al., 2015), some showing negative associations (error monitoring: Zhang et al., 2015), some showing no association (working memory, attention switching and cognitive inhibition: Franz et al., 2011; working memory: Ennis et al., 2016; Hidalgo et al., 2016) or a more complex U-shaped relationship (working memory: Moriarty et al., 2014).

The primary aims of the current study were to investigate the association of the CAR with both retrospective self-report ELS exposure and with current EF performance (working memory, cognitive flexibility and planning/problem solving) in a healthy adult male population. As gender differences have been reported in the effect of stress and ELS on HPA-axis function (DeSantis et al., 2011; Kudielka and Kirschbaum, 2005) female participants were not recruited. Due to the limited and divergent findings in prior research investigating the association between CAR magnitude and ELS in healthy adults (e.g. Engert et al., 2011; Meinlschmidt and Heim, 2005) and the mixed findings with the association between CAR and EF (see above) directional hypotheses were not made. Instead, it was hypothesised that experiencing ELS would be associated with an altered CAR and that the CAR would be associated with EF performance. The secondary aim of the current study was to investigate how 
cumulative exposure (the number of trauma types reported), the type of ELS experienced, and the developmental timing of ELS may impact on the CAR. Based on previous research it was hypothesised that both cumulative ELS exposure and experience of ELS at a younger age would be associated with a more pronounced impact on CAR magnitude. In addition, given previous findings regarding exposure to physical and sexual abuse and HPA-axis dysfunction, it was hypothesised that these types of ELS would be associated with HPA-axis function, as measured by the CAR, when controlling for other types of ELS.

\section{Material and Methods}

\section{Participants}

One hundred and nine healthy adult males (mean age: 34.2 years, SD: 10.6, range: 21-63) from a community sample participated in this research. Participants included were from the first wave of recruitment into a larger study investigating independent and interactive gene and ELS effects on EF (Klaus et al., 2017) from whom we collected saliva samples for cortisol analyses. Participants who self-reported use of steroid based medication or having a current psychiatric diagnosis or a drug or alcohol problem were excluded. There were no additional inclusion/exclusion criteria from the larger study. All participants were assessed for symptoms of psychopathology using the Hospital Anxiety and Depression Scale (HADS; Zigmond and Snaith, 1983) and for current stress levels using a 14-item Perceived Stress Scale (PSS-14; Cohen et al., 1983). Participants had normal to mild levels of anxiety, depression and current stress (means: 6.5 (SD: 3.8), 3.3 (SD: 2.6) and 20.8 (S.D. 8.2) respectively). Participants also completed questionnaires regarding their psychiatric history, demographic information (age, ethnicity and education) and sleep history (waking times and sleep durations). Twelve participants self-reported previous anxiety/depression symptoms and these participants were not disproportionately distributed when grouping participants by previous exposure to ELS. Participants were $94.5 \%$ Caucasian and $47.7 \%$ held a University level qualification. All participants gave written informed 
consent to take part and the study was approved by the School of Psychology Research Ethics Committee at the University of Lincoln.

\section{Salivary cortisol collection and measurement}

Participants were provided with 4 Salivettes (Sarstedt Ltd., Leicester, UK) for saliva collection and were instructed to wake at their usual times and to collect saliva samples immediately upon awakening and again at 30 minutes post-awakening on two consecutive week days. Participants were further instructed not to eat, drink, smoke or brush their teeth before providing samples. Across all participants mean second sample collection time was recorded as 30.23 minutes (SD: 2.02 minutes) after waking with none later than 45 minutes post-waking (max: 38 mins) and five cases prior to 30 minutes. Significant effects were unchanged when analyses were repeated after removing these five participants and so we report analyses for all participants. In order to evaluate adherence to waking sampling a subset of participants ( $\mathrm{n}=13$, approximately $12 \%$ of the total sample) wore an electronic monitoring device (Actiwatch 2; Philips Respironics, OR, USA). Across both sampling days electronically monitored waking times (mean: 07:14hrs) were not significantly different from participant recorded sampling times (mean: 07:15hrs, range: 9 minutes earlier to 12 minutes later; $\mathrm{t}(12)=1.81, \mathrm{p}=0.096$ ).

Once returned, saliva samples were stored at $-20^{\circ} \mathrm{C}$ before being made acellular through centrifugation (716G for 5 minutes (Beckman Coulter Allegra X-15R) followed by $13.3 \mathrm{G}$ for 10 minutes at $4^{\circ} \mathrm{C}$ (Eppendorf Centrifuge 5430R)) and then stored in $1.5 \mathrm{ml}$ aliquots at $-80^{\circ} \mathrm{C}$. Quantification of cortisol was determined by immunoassay (ENZO Life Sciences (UK) Ltd., Exeter, UK) conducted according to manufacturer's instructions. Assay detection limit was $0.16 \mathrm{nmol} / \mathrm{L}$ and intra and inter assay coefficients of variation were $<10 \%$ and $<18 \%$ respectively. Samples were analysed blind to whether participants had experienced ELS and thus were randomised across plates. Samples were assayed in duplicate and the CAR for day 1 and day 2 was calculated by subtracting the mean waking cortisol concentration from the mean 30 minute post-awakening concentration. The CAR from day 1 and day 2 did not significantly differ $(\mathrm{t}(108)=0.25, \mathrm{p}=0.807)$ and consequently an overall mean CAR was calculated for each participant. 


\section{Executive function}

Participants completed a battery of computerised cognitive tests from the Cambridge Neuropsychological Test Automated Battery (CANTAB; Cambridge Cognition, Cambridge, UK) described elsewhere (Klaus et al., 2017). Problem solving/planning was assessed with the One Touch Stockings of Cambridge task and was indexed by the number of problems solved on first choice. Cognitive flexibility was assessed with the Intra-Extra Dimensional Set Shift task and was indexed by the total number of errors (adjusted for number of stages completed). Working memory was assessed with the Spatial Working Memory task and was indexed by the total number of times participants revisited a box in which a token had previously been found. Further details about these EF tasks are available at http://www.cambridgecognition.com.

\section{Early life stress}

ELS was assessed using the Childhood Traumatic Events Scale (CTES; Pennebaker and Susman, 1988), which asks participants retrospectively about the occurrence of six categories of trauma: death of a close friend or relative, parental separation or divorce, traumatic sexual experience, physical violence, major illnesses or injuries, or other traumatic experiences prior to the age of 17. Participants recorded the age at which any of these traumatic experiences occurred and also rated the severity of the traumatic event on a 7-point scale (1-7) where 7 is extremely traumatic. As in Pennebaker and Susman (1988), participants had to rate an experience as 6 or 7 on the trauma scale in order to be classified as having experienced ELS.

\section{Data analysis}

All data were analysed using SPSS version 21 (IBM Corp., Armonk, NY, USA). To investigate the primary aim of the impact of experiencing ELS on the CAR, group differences (ELS vs no ELS) in CAR were investigated with one-way analysis of covariance (ANCOVA). Awakening cortisol levels, waking time, sleep duration, age and current perceived stress levels were entered as covariates as previous research suggests that these factors can impact on the CAR (Chida and Steptoe, 2009; Stalder 
et al., 2016). HADS scores were not included as covariates as they were highly correlated with scores on the PSS-14 (anxiety subscale: $\mathrm{r}=0.73, \mathrm{p}<0.001$ and depression subscale: $\mathrm{r}=0.57, \mathrm{p}<0.001$ ). Following this, secondary analyses were performed to investigate the association of the CAR with three ELS-related factors (cumulative exposure, type of stressor and developmental timing of ELS). To assess the cumulative impact of exposure to ELS we investigated how the number of trauma types experienced affected the CAR. Participants were first grouped based on the number of trauma types they reported having experienced (none, one and two or more) and then group differences were investigated using ANCOVA with covariates as before. To investigate the association between type of stressor and the CAR, the trauma types asked about in the CTES were first coded as experienced or not experienced (1 or 0$)$. Subsequently multiple linear regression analysis was performed with the CAR set as the dependent variable and the previous covariates and the coded experience data entered as independent predictors. Experience of ELS is unlikely to be an isolated event (Finkelhor et al., 2007) as such different traumas may be interrelated leading to multicollinearity within this model which can potentially bias coefficient estimates. Variance inflation factors (VIF) were examined to estimate multicollinearity severity within this model. The impact of the developmental timing of ELS was investigated by grouping participants into three groups based on the youngest age that they reported as having experienced ELS on the CTES (no ELS, childhood ELS i.e. $\leq 12$ years, adolescent ELS i.e. $\geq$ 13 years; Maercker et al., 2004). Group differences in CAR were then investigated with ANCOVA using the covariates listed previously. The age at which ELS occurred was not recorded for one participant and so this analysis was performed on 108 participants. Finally to investigate the association of the CAR with EF, three multiple linear regression analyses were performed with EF performance (working memory, cognitive flexibility and problem solving/planning) set as the dependent variable and the covariates listed previously and the CAR entered as independent predictors.

For ANCOVA and regression analyses normality of the residuals were checked by visually inspecting histograms and Q-Q plots and by checking skew and kurtosis (Kim, 2013). There was one instance of excessive deviation from a normal distribution (where errors on the cognitive flexibility task were the dependent variable) and so log transformed errors were used instead which improved the distribution 
of the residuals. Cortisol data was inspected for outliers by checking whether participant waking cortisol levels fell outside of a range (2.6-42.8 nmol/L) reported from 8am sampling in 195 men and women (Aardal and Holm, 1995). Data from three participants fell slightly outside of this range (1.89, 2.41 and $2.52 \mathrm{nmol} / \mathrm{L}$ ) however significant effects were unchanged when analyses were repeated after removing these participants and so analyses are reported for all participants. CAR can have high interindividual variability (Adam et al., 2010) however all CAR values fell within the mean \pm 4 standard deviations (as in Carnegie et al., 2014). All statistical tests were two-tailed with alpha set at 0.05. Effect sizes were calculated for all statistically significant findings using Cohen's $\mathrm{f}^{2}$, partial $\eta^{2}$ and Cohen's $d$ for regression, ANCOVA and post-hoc t-tests respectively.

\section{Results}

\section{Descriptive data}

Participants who reported experience of ELS did not significantly differ from those reporting no ELS in terms of age, years of education, waking time, sleep duration and awakening cortisol characteristics. Those who reported ELS had significantly higher HADS and PSS-14 scores (see Table 1). Across all participants cortisol levels at 30 minutes post-awakening (mean: $9.11 \mathrm{nmol} / \mathrm{L}$, S.D. 5.29) were significantly greater than at awakening (mean: $7.40 \mathrm{nmol} / \mathrm{L}$, S.D. $4.26 ; \mathrm{t}(108)=-4.27, \mathrm{p}<0.001$, Cohen's $\mathrm{d}=-0.41$ ). The overall mean CAR was $1.71 \mathrm{nmol} / \mathrm{L}$ (S.D. 4.18; 95\% CI: 0.92-2.51).

[Insert Table 1 near here]

\section{Cortisol awakening response and experience of early life stress}

The CAR was significantly elevated in those that reported experiencing ELS compared with those who did not after controlling for awakening cortisol, waking time, sleep duration, age and current perceived stress $\left(F(1,102)=7.48, p=0.007\right.$, partial $\eta^{2}=0.07 ;$ see Figure 1). 


\section{Cortisol awakening response and early life stress-related factors}

\section{Cumulative Exposure}

Across all participants, the number of trauma types experienced ranged from 0 to 3 . There was a significant difference in CAR when comparing groups based on the number of reported trauma types experienced after controlling for awakening cortisol, waking time, sleep duration, age and current perceived stress $\left(\mathrm{F}(2,101)=3.82, \mathrm{p}=0.025\right.$, partial $\left.\eta^{2}=0.07\right)$. More specifically, a stepped increase in CAR could be observed as the number of trauma types experienced increased (see Figure 2a). Posthoc t-tests indicated that the CAR was significantly elevated in those reporting having experienced one $(\mathrm{t}(92)=-2.05, \mathrm{p}=0.044$, Cohen's $\mathrm{d}=0.45)$ and those reporting having experienced two or more $(\mathrm{t}(81)$ $=-2.61, \mathrm{p}=0.011$, Cohen's $\mathrm{d}=0.66)$ trauma types compared to those reporting none.

\section{Type of early life stress}

Multiple linear regression investigating the association of CAR with type of ELS experienced showed that the full model predicted $13.7 \%$ of the variability in the CAR (adjusted $\mathrm{R}^{2}=0.137, \mathrm{~F}(11,97)=2.56$, $\mathrm{p}=0.007$, Cohen's $\mathrm{f}^{2}=0.16$ ). More specifically, experiencing physical abuse or violence was significantly positively associated with the CAR $(\mathrm{t}(97)=2.87, \mathrm{p}=0.005$; see Table 2$)$ after controlling for awakening cortisol, waking time, sleep duration, age, current perceived stress and other types of ELS. Change in adjusted $\mathrm{R}^{2}$ when the physical abuse/violence term was added to the rest of the model was 0.064 suggesting a higher than chance probability that physical abuse/violence uniquely predicted $6.4 \%$ of the variance in the CAR. None of the other ELS types were significant independent predictors of the CAR. VIF for each type of ELS in the model (range: 1.05-1.25) suggests that multicollinearity is low and unlikely to be biasing coefficient estimates. Awakening cortisol and waking time were also significant independent predictors of the CAR in this model, both having negative associations, when adjusting for the other control variables $(\mathrm{p}=0.010$ and $\mathrm{p}=0.029$ respectively; Table 2$)$.

\section{Age of early life stress}


There was a significant difference in CAR when comparing groups based on age of youngest reported experience of ELS after controlling for awakening cortisol, waking time, sleep duration, age and current perceived stress $\left(F(2,100)=3.85, p=0.024\right.$, partial $\left.\eta^{2}=0.07\right)$. Post-hoc t-tests showed that CAR was significantly elevated in those reporting experiencing younger ELS (up to 12 years) compared to those reporting no ELS $(t(92)=-2.74, p=0.007$, Cohen's $d=0.59)$ with no other significant differences between the groups (see Figure $2 b$ ).

[Insert Figure 2 and Table 2 close together and near here]

\section{Cortisol awakening response and executive function}

Multiple linear regression analyses investigating the association of the CAR with EF showed that the CAR was a significant independent predictor of planning/problem solving. More specifically CAR was significantly negatively associated with the number of problems solved on first choice on the One Touch Stockings of Cambridge task when controlling for awakening cortisol, waking time, sleep duration, age and current perceived stress $(\mathrm{t}(102)=-2.02, \mathrm{p}=0.046$; see Table 3$)$. This suggests that an elevated CAR predicted poorer planning/problem solving. The overall model was non-significant (adjusted $\mathrm{R}^{2}$ $=0.026, F(6,102)=1.48, \mathrm{p}=0.194)$ and the change in adjusted $\mathrm{R}^{2}$ when the CAR term was added to the rest of the model was 0.03 suggesting a higher than chance probability that the CAR uniquely predicted 3\% of the variance in planning/problem solving performance. Figure 3 shows the scatter plot of the bivariate correlation between CAR and problems solved on first choice unadjusted for the control variables $(p=0.027, r=-0.21)$. The CAR was not an independent predictor of the other EF indices ( $p$ values $>0.05$ ) and the overall models were also non-significant (working memory: adjusted $R^{2}=0.003$, $\mathrm{F}(6,102)=1.05, \mathrm{p}=0.400$; cognitive flexibility (log transformed): adjusted $\mathrm{R}^{2}=-0.010, \mathrm{~F}(6,102)=$ $0.82, \mathrm{p}=0.559)$.

[Insert Figure 3 and Table 3 close together and near here]

\section{Discussion}


This study investigated the relationship between the CAR and ELS and the influence of the CAR on EF in a population of healthy adult males. In line with our hypotheses, results showed a significantly elevated CAR in those reporting ELS and an elevated CAR was significantly negatively associated with planning/problem solving even after controlling for awakening cortisol levels, waking time, sleep duration, age and current perceived stress. Furthermore results showed the CAR to be associated with multiple ELS-related factors. This implies that the CAR may be an indicator of ELS-exposure and subsequent risks related to such exposure. Specifically and in line with predictions, an elevated CAR was found in those reporting both single and multiple trauma types compared to no trauma. There was a stepped increase in mean CAR from single to multiple trauma type exposure but this increase was not significant. A significantly elevated CAR was found in individuals reporting ELS in childhood but not in adolescence. Furthermore physical abuse/violence was a significant positive predictor of adult CAR and was the only stressor type uniquely associated with the CAR in this dataset.

An elevated CAR has previously been found in healthy adults reporting ELS (Engert et al., 2011) and the presence of elevated CAR is suggestive of dysregulated HPA-axis activity. However the opposite finding has also been found (Meinlschmidt and Heim, 2005) suggesting that carefully designed studies should be conducted to elucidate the reasons behind the reported discrepancies. Previous research has found positive associations between the CAR and stress perception and real world stress reactivity (Chida and Steptoe, 2009; Inslicht et al., 2011) but not laboratory-induced stress reactivity (Kidd et al., 2014). Other research suggests that an elevated CAR may be associated with vulnerability towards developing mental health disorders such as depression (Adam et al., 2010).

In concordance with the allostatic load hypothesis, which predicts that chronic or repeated exposure to stress leads to dysregulation of the neuroendocrine stress system (McEwen, 1998), previous research suggests that cumulative exposure to ELS can result in HPA-axis dysregulation. For example, postpartum women who reported experiencing both parental loss and maltreatment had elevated morning cortisol levels compared to those who experienced only one form of early adversity, or who reported no early adversity (Gonzalez et al., 2009). In addition, an elevated CAR was found in children 
(mean age 12.8 years) with moderate cumulative ELS exposure, while a similar CAR to that of a nonexposed group was found in those with higher cumulative ELS exposure ( 3 or more adversities; Gustafsson et al., 2010). This later finding has been interpreted as the early signs of HPA-axis downregulation. Our finding of a stepped increase in CAR with cumulative ELS exposure also suggests that there may be additive effects of cumulative exposure on HPA-axis functioning. However, our finding differs to that of Gustafsson and colleagues in that those with the greatest cumulative exposure had an elevated CAR compared to no exposure. It is difficult to make direct comparisons between the two studies as they have indexed cumulative exposure differently (total number of adversities versus number of trauma types experienced). The study populations also differed with regard to age (children versus adults) and recency of ELS exposure. However, it is plausible that these discrepant findings are due to heterogeneity within our highest cumulative exposure group. This group contained individuals that experienced 2 or 3 types of trauma but the actual number of traumatic events may have varied quite markedly. Further, given that this study was conducted in healthy adults there may also be within group variability in HPA-axis sensitivity/resilience to changes following experience of ELS which may become more apparent at higher levels of cumulative exposure. This is only speculation and would require follow up studies in individuals with varying experience of cumulative adversity. However the current study does find the largest standard deviation in CAR data in the group with greatest cumulative exposure (5.05 versus 3.64 and 4.49 for the no ELS and one ELS exposure groups respectively) which might support this heterogeneity argument.

It is widely understood that there are key developmental periods through which the experience of ELS may have greater or lesser effects on the developing brain (De Bellis et al., 1999) but few studies have investigated whether the developmental timing of ELS is related to subsequent HPA-axis function. It has been shown for example, that parent-reported trauma exposure before age 1 year was related to delayed cortisol recovery after acute stress in adolescents (aged 9-16 years) whereas exposure after age 1 year was related to a diverging diurnal cortisol slope (Kuhlman et al., 2015b). This suggests that age of first trauma is associated with differences in HPA-axis function in adolescence. Our finding of significantly elevated CAR in individuals who reported experiencing a traumatic life event in childhood 
( $\leq 12$ years) but not in adolescence ( $\geq 13$ years), compared to those reporting no ELS provides further support for the idea that experiencing ELS at different periods of development can lead to differential HPA-axis outcomes. Furthermore it supports wider research linking earlier exposure with negative outcomes. For instance, using the same age grouping as the present study, Maercker and colleagues (2004) found that the conditional risk for depression was more than 3.5 times greater in women reporting ELS in childhood compared with those reporting ELS in adolescence. Indeed, in general it has been found that there are poorer health outcomes if ELS is experienced at a younger age (e.g. Cicchetti et al., 2010; Enoch, 2011; Williams et al., 2016) and this is in line with other research suggesting that there may be a stress-sensitive period in amygdala development which peaks at 10 to 11 years of age (Pechtel et al., 2014) and that pre-pubertal children may be more sensitive than adolescents to negative feedback mechanisms that control cortisol output (De Bellis and Zisk, 2014).

The additional finding that physical abuse/violence was a significant positive predictor of the CAR is interesting given that previous research has shown altered HPA-axis function in those who have previously experienced physical abuse (Cicchetti et al., 2010; Carpenter et al., 2011; Kuhlman et al., 2015a). The finding is also interesting in the context of wider research on biological changes in response to this type of ELS and may be relevant to conferring a vulnerability to developing psychopathology. More specifically, childhood physical maltreatment has previously been linked to epigenetic changes in the glucocorticoid receptor gene (Romens et al., 2015) and childhood physical abuse is associated with adult stress-induced changes in blood pressure and brain activity in an established stress-sensitive neural circuit (Banihashemi et al., 2015). Epidemiological studies, narrative and meta-analytic reviews suggest that physical abuse is associated with depression and other psychiatric disorders (Barter and Stanley, 2016; Carr et al., 2013; Mandelli et al., 2015). However, other types of ELS are often found to be better predictors of poor mental health outcomes (Mandelli et al., 2015; Sheikh et al., 2016). For example Mandelli and colleagues found that sexual abuse was more strongly associated with depression than domestic violence and physical abuse. It should be noted that endorsement for some types of stress, including sexual abuse, was very low (see Table 2) which may have limited the ability to detect significant effects in these trauma types. However, as the current study 
investigated the association between different types of stressor and the CAR in males, it is possible that gender may impact upon these relationships. Gender differences in brain development following different types of ELS have been reported (Teicher et al., 2004) in addition to gender differences in neural activation during executive and affective processing subsequent to ELS (Crozier et al., 2014). The CTES was used to identify trauma in the current study and although this measure contains an 'other' category, it does not include traumas related to neglect which may have resulted in the identification of associations between the CAR and this type of ELS as reported previously (Peng et al., 2014).

A significant negative association between the CAR and problems solved on first choice in the One Touch Stockings of Cambridge task was found, suggesting that an elevated CAR may be associated with poorer problem solving/planning. There was no significant association between the CAR and working memory or cognitive flexibility. This is the first study to investigate the association between the CAR and problem solving/planning in a healthy adult sample and this finding is interesting when considered in the context of other research. Firstly, working memory and cognitive flexibility are considered two of the three core EFs (along with inhibitory control not assessed here) and problem solving/planning are considered higher-order EFs that are 'built' from these core EFs (Diamond, 2013). Small, non-significant effects with core EFs may be additive and lead to larger effects in higher-order EFs and this could be tested experimentally by taxing specific core aspects of EF in order to see how this effects the association of the CAR with higher-order EF. Secondly, planning is an aspect of future oriented thinking linked to consideration of future consequences. Previous research suggests that witnessing or being a victim of violence during adolescence may be linked to poor development of future orientation (Monahan et al., 2015) and this research is particularly relevant in light of the physical abuse/violence and CAR association finding in the current study. Previous research has also found a similar negative association between CAR and error monitoring (Zhang et al., 2015). Error monitoring, an important aspect of adaptive cognition, allows for behavioural adjustments that prevent future mistakes in goal-directed action via the implementation of cognitive control (Alexander and Brown, 2010). Zhang and colleagues found that an elevated CAR was associated with poorer monitoring of errors and reduced behavioural adjustment following error. This is interesting given that planning and 
error monitoring are both subserved by the prefrontal cortex and both have a future-oriented perspective i.e. are important cognitive functions for goal pursuit (Fuster and Bressler, 2015).

Importantly our findings are correlational and so causality cannot be inferred. There are common brain regions involved in both EF and the regulation of HPA-axis activity (e.g. Boehringer et al., 2015). However, stress-induced dysregulation of the HPA-axis can lead to increased glucocorticoid release (Gunnar and Quevedo, 2007) which can be neurotoxic and impair neural plasticity in sensitive brain regions (Lupien et al., 2009; Teicher et al., 2003). The prefrontal cortex is a region known to have a protracted development and a high concentration of glucocorticoid receptors and thus is considered to be particularly sensitive to structural brain changes induced by chronic stress (e.g. Brown et al., 2005; Izquierdo et al., 2006). This stress can also lead to functional changes in prefrontal catecholamine pathways e.g. increased noradrenergic and reduced dopamine transmission (Arnsten, 2009). These structural and functional brain changes can potentially impact on EF in adulthood to cause difficulties in problem solving/planning as observed in this study. Planning has also been associated with the cerebellum (Bauer et al., 2009) which is also known to have a particularly protracted development (Tiemeier et al., 2010). This may leave the cerebellum susceptible to stress-induced brain changes that could impact higher-order cerebella-subserved cognitive functions such as planning. In a larger but overlapping sample of participants to the current study, we have recently reported poorer problem solving/planning in individuals with a genotype previously shown to be associated with reduced striatal dopamine D2 receptor availability (Klaus et al., 2017). Unfortunately we do not have cortisol data for the larger sample but future work should use larger sample sizes to enable grouping based on both experience of ELS and genotype. This would enable researchers to investigate whether genetic differences, in systems known to be vulnerable to ELS-induced alterations, interact with HPA-axis function to confer greater risk for mental health disorders or risk factors for such disorders.

Our CAR and problem solving/planning finding is interesting given that an elevated CAR may confer vulnerability for developing mental health problems such as depression (Adam et al., 2010). Poorer problem solving/planning may represent a cognitive mechanism by which an elevated CAR increases 
depression risk. A number of studies have found evidence of problem solving/planning impairments in depressed patients (Fossati et al., 2002). These impairments may be a component of a cognitive pathway leading to suicidal behaviour (Roskar et al., 2007) but importantly they may pre-date the onset of depression (Knouse et al., 2013). For example Knouse et al., (2013) show that self-management of time, self-organization, and problem solving were related to the risk of depression development. Interpretation of our EF findings should be made with caution however. Three separate regression analyses were performed (for working memory, cognitive flexibility and problem solving/planning) this may increase the chance of a type I error. Future work should aim to replicate our novel finding and to then establish the mechanisms behind the CAR and problem solving/planning association.

There were some limitations in the current study. Previous studies with women have typically reported elevated CAR compared to men (Pruessner et al., 1997; Vreeburg et al., 2009; Wust et al., 2000) and this may explain the relatively low mean CAR $(1.71 \mathrm{nmol} / \mathrm{L})$ found in the current study. Alternatively low mean CAR may result from late awakening times given the significant association between waking time and CAR in this and other studies (e.g. Kudielka and Kirschbaum, 2003) and the fact that waking time was not dictated in the study design. Low CAR might also be due to not having recorded the full peak of the cortisol rise. This study was designed prior to consensus guidelines suggesting that saliva collection at 45 and 60 minutes reduces the chance of missing the full peak (Stalder et al., 2016). However, many previous reports have used a 0, 30 sampling design (e.g. Corbett and Schupp, 2014). In addition, our waking sample compliance data suggested no significant delay in taking samples (mean: 1 min delay) and awakening cortisol and waking time were significantly negatively associated with the CAR as have previously been reported (Kudielka and Kirschbaum, 2003; Wust et al., 2000) providing confidence in the sampling methodology used in the current study. Nevertheless, future work should refer to Stalder and colleagues consensus guidelines regarding methodology. Future work should also consider additional factors that might influence the CAR which were not taken into consideration and could potentially bias conclusions of the current study such as habitual smoking, body mass index and ambient light levels (see Stalder et al., (2016) for an overview). 
In this study, current and previous psychiatric health of participants were probed using self-report assessments, future work could instead provide an objective, potentially less biased clinical assessment. An additional potential limitation of this study was the measure used to evaluate childhood trauma. The CTES (Pennebaker and Susman, 1988) precluded an analysis based on the number of events (hence we use number of trauma types experienced as the cumulative measure) and was retrospective and subjective in nature which may have resulted in biases in reporting. However having participants rate the trauma level of events could be considered an advantage of this measure over a checklist based approach. Another limitation is the all-male sample which may limit the generalization of our results and future work should include female participants and consider stratified analyses. Notwithstanding these limitations, the fact that this study showed that a number of ELS-related factors influenced the CAR in a manner that is consistent with previous research provides support for our finding that elevated CAR is associated with ELS in this population.

\section{Conclusions}

This study found that elevated CAR was associated with self-reported experience of ELS in a healthy male population and is the first study in which to report this effect in the context of cumulative exposure, developmental timing of ELS and type of stressor within the same study population. These findings suggest that individuals who have experienced ELS in childhood, as opposed to adolescence, who have experienced physical abuse/violence and who have experienced cumulative ELS are at risk of having an elevated cortisol awakening response and potentially altered chronic functioning of their HPA axis. In addition a significant negative association was found between the CAR and problem solving/planning in the same population which provides evidence that an elevated CAR, possibly in response to ELS, may be a risk phenotype for the future development of mental health disorders rather than a sign of resilience in healthy adult males. Future work delineating further the combined risk of elevated CAR and poor problem solving/planning in the context of ELS is warranted. This could include taking additional measures of HPA-axis function (such as plasma adrenocorticotrophic hormone, corticotrophin releasing factor or diurnal cortisol levels) or using prospective follow-up studies to see how well the combined phenotype predicts subsequent development of mood or anxiety 
disorders. As we get closer to elucidating individual risk phenotypes of mental health disorders the hope is that we get closer to more tailored and efficacious health care and a greater understanding of the impact of environmental events, at key stages of development, on brain health.

\section{Acknowledgments}

We thank Dr Simon Durrant from the School of Psychology at the University of Lincoln, for the loan of Actiwatches that were used in this study and for his invaluable comments and advice. We also thank

Dr Daniel Wenman and Jake Leyhr for their research assistance with the project and Dr Humberto Gutierrez, Dr Julian Bartrup and Dr William Hayes in the School of Life Sciences at the University of Lincoln for their help and advice with the cortisol analysis work.

\section{Funding}

This work was supported by the University of Lincoln's School of Psychology, the College of Social Science Research Fund and the Academic Return to Research Fund $\left(R^{2} F\right)$ all awarded to KP.

\section{References}

Aardal, E. \& Holm, A. C. 1995. Cortisol in saliva--reference ranges and relation to cortisol in serum. Eur J Clin Chem Clin Biochem, 33, 927-32.

Adam, E. K., Doane, L. D., Zinbarg, R. E., Mineka, S., Craske, M. G. \& Griffith, J. W. 2010. Prospective prediction of major depressive disorder from cortisol awakening responses in adolescence. Psychoneuroendocrinology, 35, 921-31.

Adam, E. K., Vrshek-Schallhorn, S., Kendall, A. D., Mineka, S., Zinbarg, R. E. \& Craske, M. G. 2014. Prospective associations between the cortisol awakening response and first onsets of anxiety disorders over a six-year follow-up--2013 Curt Richter Award Winner. Psychoneuroendocrinology, 44, 47-59.

Alexander, W. H. \& Brown, J. W. 2010. Computational models of performance monitoring and cognitive control. Top Cogn Sci, 2, 658-77.

Almasy, L. \& Blangero, J. 2001. Endophenotypes as quantitative risk factors for psychiatric disease: rationale and study design. Am J Med Genet, 105, 42-4.

Almela, M., van der Meij, L., Hidalgo, V., Villada, C. \& Salvador, A. 2012. The cortisol awakening response and memory performance in older men and women. Psychoneuroendocrinology, 37, 1929-40.

Arnsten, A. F. 2009. Stress signalling pathways that impair prefrontal cortex structure and function. Nat Rev Neurosci, 10, 410-22.

Banihashemi, L., Sheu, L. K., Midei, A. J. \& Gianaros, P. J. 2015. Childhood physical abuse predicts stressor-evoked activity within central visceral control regions. Soc Cogn Affect Neurosci, 10, 474-85. 
Barter, C. \& Stanley, N. 2016. Inter-personal violence and abuse in adolescent intimate relationships: mental health impact and implications for practice. Int Rev Psychiatry, 1-19.

Bauer, P. M., Hanson, J. L., Pierson, R. K., Davidson, R. J. \& Pollak, S. D. 2009. Cerebellar volume and cognitive functioning in children who experienced early deprivation. Biol Psychiatry, 66, 11006.

Berger, M., Kraeuter, A. K., Romanik, D., Malouf, P., Amminger, G. P. \& Sarnyai, Z. 2016. Cortisol awakening response in patients with psychosis: Systematic review and meta-analysis. Neurosci Biobehav Rev, 68, 157-66.

Boehringer, A., Tost, H., Haddad, L., Lederbogen, F., Wust, S., Schwarz, E. \& Meyer-Lindenberg, A. 2015. Neural Correlates of the Cortisol Awakening Response in Humans. Neuropsychopharmacology, 40, 2278-85.

Bos, K. J., Fox, N., Zeanah, C. H. \& Nelson III, C. A. 2009. Effects of early psychosocial deprivation on the development of memory and executive function. Front Behav Neurosci, 3, 16.

Brown, S. M., Henning, S. \& Wellman, C. L. 2005. Mild, short-term stress alters dendritic morphology in rat medial prefrontal cortex. Cereb Cortex, 15, 1714-22.

Cardona, J. F., Manes, F., Escobar, J., Lopez, J. \& Ibanez, A. 2012. Potential consequences of abandonment in preschool-age: neuropsychological findings in institutionalized children. Behav Neurol, 25, 291-301.

Carnegie, R., Araya, R., Ben-Shlomo, Y., Glover, V., O'Connor, T. G., O'Donnell, K. J., Pearson, R. \& Lewis, G. 2014. Cortisol awakening response and subsequent depression: prospective longitudinal study. Br J Psychiatry, 204, 137-43.

Carpenter, L. L., Shattuck, T. T., Tyrka, A. R., Geracioti, T. D. \& Price, L. H. 2011. Effect of childhood physical abuse on cortisol stress response. Psychopharmacology (Berl), 214, 367-75.

Carr, C. P., Martins, C. M., Stingel, A. M., Lemgruber, V. B. \& Juruena, M. F. 2013. The role of early life stress in adult psychiatric disorders: a systematic review according to childhood trauma subtypes. J Nerv Ment Dis, 201, 1007-20.

Chida, Y. \& Steptoe, A. 2009. Cortisol awakening response and psychosocial factors: a systematic review and meta-analysis. Biol Psychol, 80, 265-78.

Cicchetti, D. \& Rogosch, F. A. 2001. Diverse patterns of neuroendocrine activity in maltreated children. Dev Psychopathol, 13, 677-93.

Cicchetti, D., Rogosch, F. A., Gunnar, M. R. \& Toth, S. L. 2010. The differential impacts of early physical and sexual abuse and internalizing problems on daytime cortisol rhythm in school-aged children. Child Dev, 81, 252-69.

Clow, A., Thorn, L., Evans, P. \& Hucklebridge, F. 2004. The awakening cortisol response: methodological issues and significance. Stress, 7, 29-37.

Cohen, S., Kamarck, T. \& Mermelstein, R. 1983. A global measure of perceived stress. J Health Soc Behav, 24, 385-96.

Corbett, B. A. \& Schupp, C. W. 2014. The cortisol awakening response (CAR) in male children with autism spectrum disorder. Horm Behav, 65, 345-50.

Crozier, J. C., Wang, L., Huettel, S. A. \& De Bellis, M. D. 2014. Neural correlates of cognitive and affective processing in maltreated youth with posttraumatic stress symptoms: does gender matter? Dev Psychopathol, 26, 491-513.

Davidovich, S., Collishaw, S., Thapar, A. K., Harold, G., Thapar, A. \& Rice, F. 2016. Do better executive functions buffer the effect of current parental depression on adolescent depressive symptoms? J Affect Disord, 199, 54-64.

De Bellis, M. D., Keshavan, M. S., Clark, D. B., Casey, B. J., Giedd, J. N., Boring, A. M., Frustaci, K. \& Ryan, N. D. 1999. A.E. Bennett Research Award. Developmental traumatology. Part II: Brain development. Biol Psychiatry, 45, 1271-84.

De Bellis, M. D. \& Zisk, A. 2014. The biological effects of childhood trauma. Child Adolesc Psychiatr Clin $N$ Am, 23, 185-222, vii. 
DeSantis, S. M., Baker, N. L., Back, S. E., Spratt, E., Ciolino, J. D., Moran-Santa Maria, M., Dipankar, B. \& Brady, K. T. 2011. Gender differences in the effect of early life trauma on hypothalamicpituitary-adrenal axis functioning. Depress Anxiety, 28, 383-92.

Diamond, A. 2013. Executive functions. Annu Rev Psychol, 64, 135-68.

Doom, J. R. \& Gunnar, M. R. 2013. Stress physiology and developmental psychopathology: past, present, and future. Dev Psychopathol, 25, 1359-73.

Engert, V., Efanov, S. I., Dedovic, K., Dagher, A. \& Pruessner, J. C. 2011. Increased cortisol awakening response and afternoon/evening cortisol output in healthy young adults with low early life parental care. Psychopharmacology (Berl), 214, 261-8.

Ennis, G. E., Moffat, S. D. \& Hertzog, C. 2016. The cortisol awakening response and cognition across the adult lifespan. Brain Cogn, 105, 66-77.

Enoch, M. A. 2011. The role of early life stress as a predictor for alcohol and drug dependence. Psychopharmacology (Berl), 214, 17-31.

Evans, P., Hucklebridge, F., Loveday, C. \& Clow, A. 2012. The cortisol awakening response is related to executive function in older age. Int J Psychophysiol, 84, 201-4.

Finkelhor, D., Ormrod, R. K. \& Turner, H. A. 2007. Poly-victimization: a neglected component in child victimization. Child Abuse Negl, 31, 7-26.

Fossati, P., Ergis, A. M. \& Allilaire, J. F. 2002. [Executive functioning in unipolar depression: a review]. Encephale, 28, 97-107.

Franz, C. E., O'Brien, R. C., Hauger, R. L., Mendoza, S. P., Panizzon, M. S., Prom-Wormley, E., Eaves, L. J., Jacobson, K., Lyons, M. J., Lupien, S., Hellhammer, D., Xian, H. \& Kremen, W. S. 2011. Crosssectional and 35-year longitudinal assessment of salivary cortisol and cognitive functioning: the Vietnam Era twin study of aging. Psychoneuroendocrinology, 36, 1040-52.

Fuster, J. M. \& Bressler, S. L. 2015. Past makes future: role of pFC in prediction. J Cogn Neurosci, 27, 639-54.

Gonzalez, A., Jenkins, J. M., Steiner, M. \& Fleming, A. S. 2009. The relation between early life adversity, cortisol awakening response and diurnal salivary cortisol levels in postpartum women. Psychoneuroendocrinology, 34, 76-86.

Gottesman, II \& Gould, T. D. 2003. The endophenotype concept in psychiatry: etymology and strategic intentions. Am J Psychiatry, 160, 636-45.

Gunnar, M. \& Quevedo, K. 2007. The neurobiology of stress and development. Annu Rev Psychol, 58, 145-73.

Gustafsson, P. E., Anckarsater, H., Lichtenstein, P., Nelson, N. \& Gustafsson, P. A. 2010. Does quantity have a quality all its own? Cumulative adversity and up- and down-regulation of circadian salivary cortisol levels in healthy children. Psychoneuroendocrinology, 35, 1410-5.

Hidalgo, V., Almela, M., Pulopulos, M. M. \& Salvador, A. 2016. Memory performance is related to the cortisol awakening response in older people, but not to the diurnal cortisol slope. Psychoneuroendocrinology, 71, 136-46.

Inslicht, S. S., Otte, C., McCaslin, S. E., Apfel, B. A., Henn-Haase, C., Metzler, T., Yehuda, R., Neylan, T. C. \& Marmar, C. R. 2011. Cortisol awakening response prospectively predicts peritraumatic and acute stress reactions in police officers. Biol Psychiatry, 70, 1055-62.

Izquierdo, A., Wellman, C. L. \& Holmes, A. 2006. Brief uncontrollable stress causes dendritic retraction in infralimbic cortex and resistance to fear extinction in mice. $J$ Neurosci, 26, 5733-8.

Kidd, T., Carvalho, L. A. \& Steptoe, A. 2014. The relationship between cortisol responses to laboratory stress and cortisol profiles in daily life. Biol Psychol, 99, 34-40.

Kim, H. Y. 2013. Statistical notes for clinical researchers: assessing normal distribution (2) using skewness and kurtosis. Restor Dent Endod, 38, 52-4.

Klaus, K., Butler, K., Durrant, S. J., Ali, M., Inglehearn, C. F., Hodgson, T. L., Gutierrez, H. \& Pennington, K. 2017. The effect of COMT Val158Met and DRD2 C957T polymorphisms on executive function and the impact of early life stress. Brain and Behavior, e00695-n/a. 
Knouse, L. E., Barkley, R. A. \& Murphy, K. R. 2013. Does executive functioning (EF) predict depression in clinic-referred adults? EF tests vs. rating scales. J Affect Disord, 145, 270-5.

Kudielka, B. M. \& Kirschbaum, C. 2003. Awakening cortisol responses are influenced by health status and awakening time but not by menstrual cycle phase. Psychoneuroendocrinology, 28, 35-47.

Kudielka, B. M. \& Kirschbaum, C. 2005. Sex differences in HPA axis responses to stress: a review. Biol Psychol, 69, 113-32.

Kuhlman, K. R., Geiss, E. G., Vargas, I. \& Lopez-Duran, N. L. 2015a. Differential associations between childhood trauma subtypes and adolescent HPA-axis functioning. Psychoneuroendocrinology, 54, 103-14.

Kuhlman, K. R., Vargas, I., Geiss, E. G. \& Lopez-Duran, N. L. 2015b. Age of Trauma Onset and HPA Axis Dysregulation Among Trauma-Exposed Youth. J Trauma Stress, 28, 572-9.

Law, R., Evans, P., Thorn, L., Hucklebridge, F. \& Clow, A. 2015. The cortisol awakening response predicts same morning executive function: results from a 50-day case study. Stress, 18, 61621.

Loman, M. M., Johnson, A. E., Westerlund, A., Pollak, S. D., Nelson, C. A. \& Gunnar, M. R. 2013. The effect of early deprivation on executive attention in middle childhood. J Child Psychol Psychiatry, 54, 37-45.

Lupien, S. J., McEwen, B. S., Gunnar, M. R. \& Heim, C. 2009. Effects of stress throughout the lifespan on the brain, behaviour and cognition. Nat Rev Neurosci, 10, 434-45.

Maercker, A., Michael, T., Fehm, L., Becker, E. S. \& Margraf, J. 2004. Age of traumatisation as a predictor of post-traumatic stress disorder or major depression in young women. $\mathrm{Br} J$ Psychiatry, 184, 482-7.

Mandelli, L., Petrelli, C. \& Serretti, A. 2015. The role of specific early trauma in adult depression: A meta-analysis of published literature. Childhood trauma and adult depression. Eur Psychiatry, 30, 665-80.

McEwen, B. S. 1998. Stress, adaptation, and disease. Allostasis and allostatic load. Ann N Y Acad Sci, 840, 33-44.

McLaughlin, K. A., Conron, K. J., Koenen, K. C. \& Gilman, S. E. 2010. Childhood adversity, adult stressful life events, and risk of past-year psychiatric disorder: a test of the stress sensitization hypothesis in a population-based sample of adults. Psychol Med, 40, 1647-58.

McTeague, L. M., Goodkind, M. S. \& Etkin, A. 2016. Transdiagnostic impairment of cognitive control in mental illness. J Psychiatr Res, 83, 37-46.

Meinlschmidt, G. \& Heim, C. 2005. Decreased cortisol awakening response after early loss experience. Psychoneuroendocrinology, 30, 568-76.

Mittal, C., Griskevicius, V., Simpson, J. A., Sung, S. \& Young, E. S. 2015. Cognitive adaptations to stressful environments: When childhood adversity enhances adult executive function. $J$ Pers Soc Psychol, 109, 604-21.

Monahan, K. C., King, K. M., Shulman, E. P., Cauffman, E. \& Chassin, L. 2015. The effects of violence exposure on the development of impulse control and future orientation across adolescence and early adulthood: Time-specific and generalized effects in a sample of juvenile offenders. Dev Psychopathol, 27, 1267-83.

Moriarty, A. S., Bradley, A. J., Anderson, K. N., Watson, S., Gallagher, P. \& McAllister-Williams, R. H. 2014. Cortisol awakening response and spatial working memory in man: a U-shaped relationship. Hum Psychopharmacol, 29, 295-8.

Pechtel, P., Lyons-Ruth, K., Anderson, C. M. \& Teicher, M. H. 2014. Sensitive periods of amygdala development: the role of maltreatment in preadolescence. Neuroimage, 97, 236-44.

Pechtel, P. \& Pizzagalli, D. A. 2011. Effects of early life stress on cognitive and affective function: an integrated review of human literature. Psychopharmacology (Berl), 214, 55-70.

Peng, H., Long, Y., Li, J., Guo, Y., Wu, H., Yang, Y., Ding, Y., He, J. \& Ning, Y. 2014. Hypothalamicpituitary-adrenal axis functioning and dysfunctional attitude in depressed patients with and without childhood neglect. BMC Psychiatry, 14, 45. 
Pennebaker, J. W. \& Susman, J. R. 1988. Disclosure of traumas and psychosomatic processes. Soc Sci Med, 26, 327-32.

Pruessner, J. C., Wolf, O. T., Hellhammer, D. H., Buske-Kirschbaum, A., von Auer, K., Jobst, S., Kaspers, F. \& Kirschbaum, C. 1997. Free cortisol levels after awakening: a reliable biological marker for the assessment of adrenocortical activity. Life Sci, 61, 2539-49.

Read, J., Perry, B. D., Moskowitz, A. \& Connolly, J. 2001. The contribution of early traumatic events to schizophrenia in some patients: a traumagenic neurodevelopmental model. Psychiatry, 64, 319-45.

Romens, S. E., McDonald, J., Svaren, J. \& Pollak, S. D. 2015. Associations between early life stress and gene methylation in children. Child Dev, 86, 303-9.

Roskar, S., Zorko, M., Bucik, V. \& Marusic, A. 2007. Problem solving for depressed suicide attempters and depressed individuals without suicide attempt. Psychiatr Danub, 19, 296-302.

Sheikh, M. A., Abelsen, B. \& Olsen, J. A. 2016. Clarifying Associations between Childhood Adversity, Social Support, Behavioral Factors, and Mental Health, Health, and Well-Being in Adulthood: A Population-Based Study. Front Psychol, 7, 727.

Stalder, T., Kirschbaum, C., Kudielka, B. M., Adam, E. K., Pruessner, J. C., Wust, S., Dockray, S., Smyth, N., Evans, P., Hellhammer, D. H., Miller, R., Wetherell, M. A., Lupien, S. J. \& Clow, A. 2016. Assessment of the cortisol awakening response: Expert consensus guidelines. Psychoneuroendocrinology, 63, 414-32.

Teicher, M. H., Andersen, S. L., Polcari, A., Anderson, C. M., Navalta, C. P. \& Kim, D. M. 2003. The neurobiological consequences of early stress and childhood maltreatment. Neurosci Biobehav Rev, 27, 33-44.

Teicher, M. H., Dumont, N. L., Ito, Y., Vaituzis, C., Giedd, J. N. \& Andersen, S. L. 2004. Childhood neglect is associated with reduced corpus callosum area. Biol Psychiatry, 56, 80-5.

Tiemeier, H., Lenroot, R. K., Greenstein, D. K., Tran, L., Pierson, R. \& Giedd, J. N. 2010. Cerebellum development during childhood and adolescence: a longitudinal morphometric MRI study. Neuroimage, 49, 63-70.

Veen, G., van Vliet, I. M., DeRijk, R. H., Giltay, E. J., van Pelt, J. \& Zitman, F. G. 2011. Basal cortisol levels in relation to dimensions and DSM-IV categories of depression and anxiety. Psychiatry Res, $185,121-8$.

Vreeburg, S. A., Kruijtzer, B. P., van Pelt, J., van Dyck, R., DeRijk, R. H., Hoogendijk, W. J., Smit, J. H., Zitman, F. G. \& Penninx, B. W. 2009. Associations between sociodemographic, sampling and health factors and various salivary cortisol indicators in a large sample without psychopathology. Psychoneuroendocrinology, 34, 1109-20.

Williams, L. M., Debattista, C., Duchemin, A. M., Schatzberg, A. F. \& Nemeroff, C. B. 2016. Childhood trauma predicts antidepressant response in adults with major depression: data from the randomized international study to predict optimized treatment for depression. Trans/ Psychiatry, 6, e799.

Wust, S., Wolf, J., Hellhammer, D. H., Federenko, I., Schommer, N. \& Kirschbaum, C. 2000. The cortisol awakening response - normal values and confounds. Noise Health, 2, 79-88.

Zhang, L., Duan, H., Qin, S., Yuan, Y., Buchanan, T. W., Zhang, K. \& Wu, J. 2015. High cortisol awakening response is associated with impaired error monitoring and decreased post-error adjustment. Stress, 18, 561-8.

Zigmond, A. S. \& Snaith, R. P. 1983. The hospital anxiety and depression scale. Acta Psychiatr Scand, $67,361-70$. 


\begin{tabular}{lccc}
\hline \multicolumn{1}{c}{ Characteristic } & $\begin{array}{c}\text { No ELS } \\
(\mathrm{n}=68)\end{array}$ & $\begin{array}{c}\text { ELS } \\
(\mathrm{n}=41)\end{array}$ & Sig. \\
\hline Age (Years) & $33.85(1.29)$ & $34.78(1.67)$ & $\mathrm{t}(107)=-0.44, \mathrm{p}=0.660$ \\
Years of Education & $14.62(0.30)$ & $14.39(0.35)$ & $\mathrm{t}(107)=0.48, \mathrm{p}=0.632$ \\
HADS Anxiety & $5.71(0.40)$ & $7.85(0.66)$ & $\mathrm{t}(107)=-2.97, \mathrm{p}=\mathbf{0 . 0 0 4 ,} \mathbf{d}=\mathbf{0 . 5 7}$ \\
HADS Depression & $2.69(0.28)$ & $4.17(0.46)$ & $\mathrm{t}(107)=-2.93, \mathbf{p}=\mathbf{0 . 0 0 4 ,} \mathbf{d}=\mathbf{0 . 5 6}$ \\
PSS-14 & $19.34(0.95)$ & $23.29(1.32)$ & $\mathrm{t}(107)=-2.49, \mathrm{p}=\mathbf{0 . 0 1 4}, \mathbf{d}=\mathbf{0 . 4 9}$ \\
Waking Time & $07: 25(0: 08)$ & $07: 38(0: 13)$ & $\mathrm{t}(107)=-0.85, \mathrm{p}=0.396$ \\
Sleep Duration (Hours) & $6.66(0.14)$ & $6.62(0.24)$ & $\mathrm{t}(65.44)=0.14, \mathrm{p}=0.883$ \\
Awakening Cortisol (nmol/L) & $7.55(0.53)$ & $7.15(0.63)$ & $\mathrm{t}(107)=0.47, \mathrm{p}=0.637$ \\
\hline
\end{tabular}

Table 1: Participant characteristics, sleep and awakening cortisol data.

Data shown are means and standard error of the mean. ELS $=$ Early life stress, HADS $=$ Hospital Anxiety and Depression Scale, PSS-14 = Perceived Stress Scale, $d=$ Cohen's $d$. 


\begin{tabular}{lccc}
\hline & $\boldsymbol{\beta}$ & $\mathbf{t}(\mathbf{9 7})$ & Sig. \\
\hline Intercept & - & 2.74 & $\mathrm{p}=0.007$ \\
Awakening cortisol & -0.24 & -2.64 & $\mathbf{p}=\mathbf{0 . 0 1 0}$ \\
Waking time & -0.23 & -2.22 & $\mathbf{p}=\mathbf{0 . 0 2 9}$ \\
Sleep duration & -0.14 & -1.46 & $\mathrm{p}=0.148$ \\
Age & -0.08 & -0.78 & $\mathrm{p}=0.439$ \\
PSS-14 & 0.05 & 0.47 & $\mathrm{p}=0.639$ \\
Death $(\mathrm{n}=10)$ & -0.06 & -0.58 & $\mathrm{p}=0.562$ \\
Divorce/Parental separation $(\mathrm{n}=12)$ & 0.07 & 0.75 & $\mathrm{p}=0.454$ \\
Sexual abuse $(\mathrm{n}=4)$ & 0.08 & 0.78 & $\mathrm{p}=0.436$ \\
Physical abuse/Violence $(\mathrm{n}=10)$ & 0.28 & 2.87 & $\mathbf{p}=\mathbf{0 . 0 0 5}$ \\
Illness/Injury $(\mathrm{n}=6)$ & 0.05 & 0.46 & $\mathrm{p}=0.648$ \\
Other ELS $(\mathrm{n}=22)$ & 0.16 & 1.61 & $\mathrm{p}=0.111$ \\
\hline
\end{tabular}

Table 2: Associations between the cortisol awakening response and different early life stressors. $\beta$ is the standardised regression coefficient, PSS-14 is the Perceived Stress Scale score. 
(C) 2017. This manuscript version is made available under the CC-BY-NC-ND 4.0 license http://creativecommons.org/licenses/by-nc-nd/4.0/

\begin{tabular}{lccc}
\hline & $\boldsymbol{\beta}$ & $\mathbf{t}(\mathbf{1 0 2})$ & $\mathbf{S i g}$. \\
\hline Intercept & - & 6.51 & $\mathrm{p}<0.001$ \\
Awakening cortisol & 0.09 & 0.89 & $\mathrm{p}=0.373$ \\
Waking time & -0.10 & -0.99 & $\mathrm{p}=0.326$ \\
Sleep duration & -0.07 & -0.74 & $\mathrm{p}=0.461$ \\
Age & -0.11 & -1.01 & $\mathrm{p}=0.317$ \\
PSS-14 & -0.12 & -1.26 & $\mathrm{p}=0.212$ \\
Cortisol awakening response & -0.20 & -2.02 & $\mathbf{p}=\mathbf{0 . 0 4 6}$ \\
\hline
\end{tabular}

Table 3: Association between problem solving/planning and the cortisol awakening response. $\beta$ is the standardised regression coefficient, PSS-14 is the Perceived Stress Scale score. 
Figure 1: Mean cortisol awakening response (CAR) for participants with and without self-reported experience of early life stress (ELS). Data show mean and standard error of the mean. ** CAR in ELS group > No ELS group, $\mathrm{p}=0.007)$.

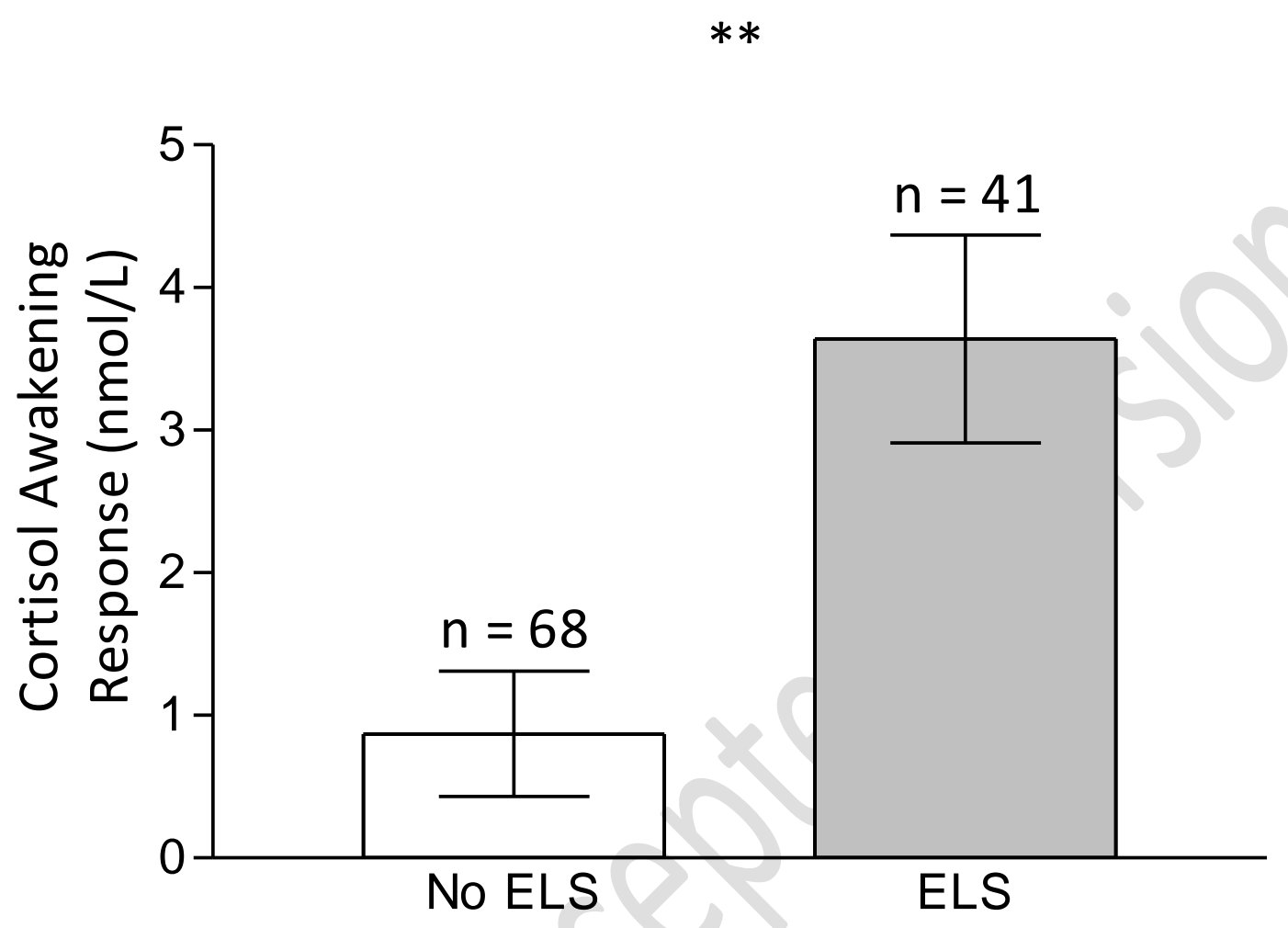


Figure 2: Impact of cumulative early life stress (ELS) exposure (2a) and developmental timing of ELS exposure (2b) on the cortisol awakening response (CAR). Data show mean and standard error of the mean. * CAR in no ELS group $<$ CAR in groups reporting experience of one and two or more types of ELS, p's $<0.05$. ** CAR in no ELS group $<$ CAR in those reporting childhood ELS, $\mathrm{p}=$ $0.007)$.

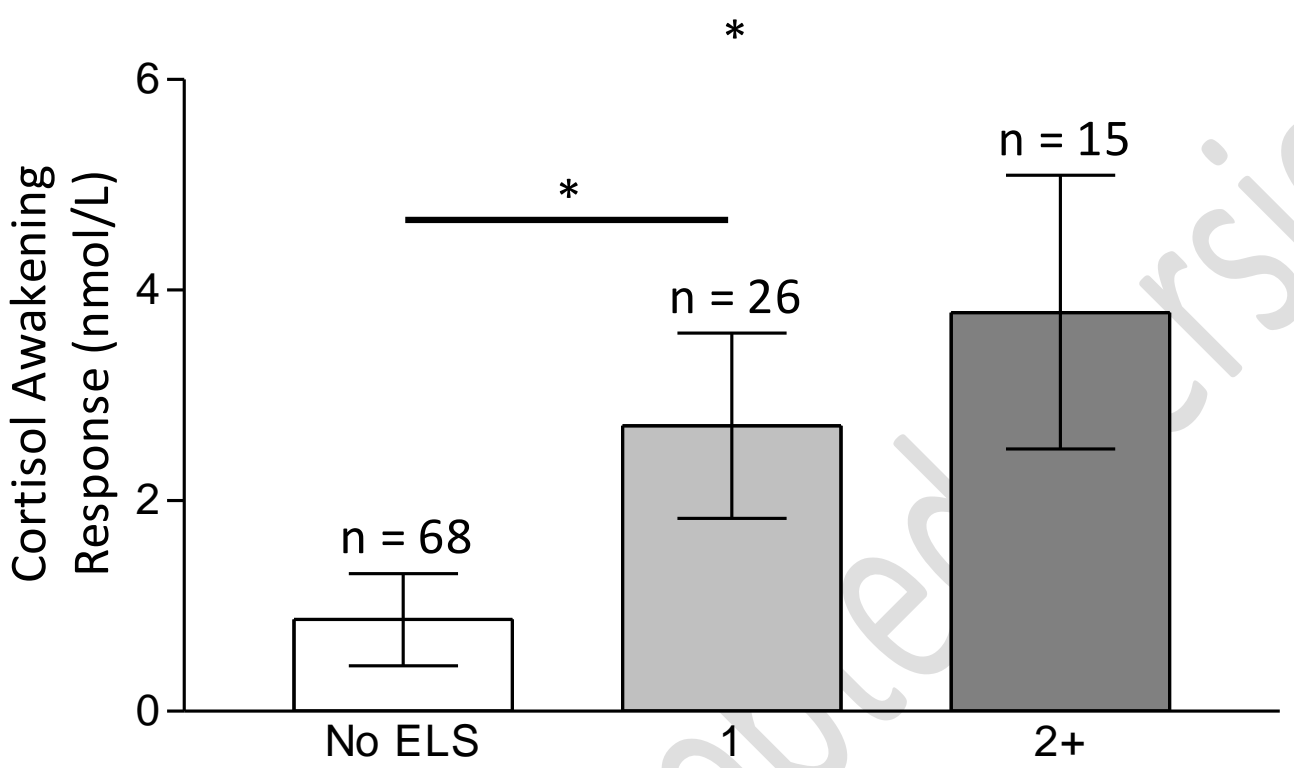

Number of Trauma Types Experienced

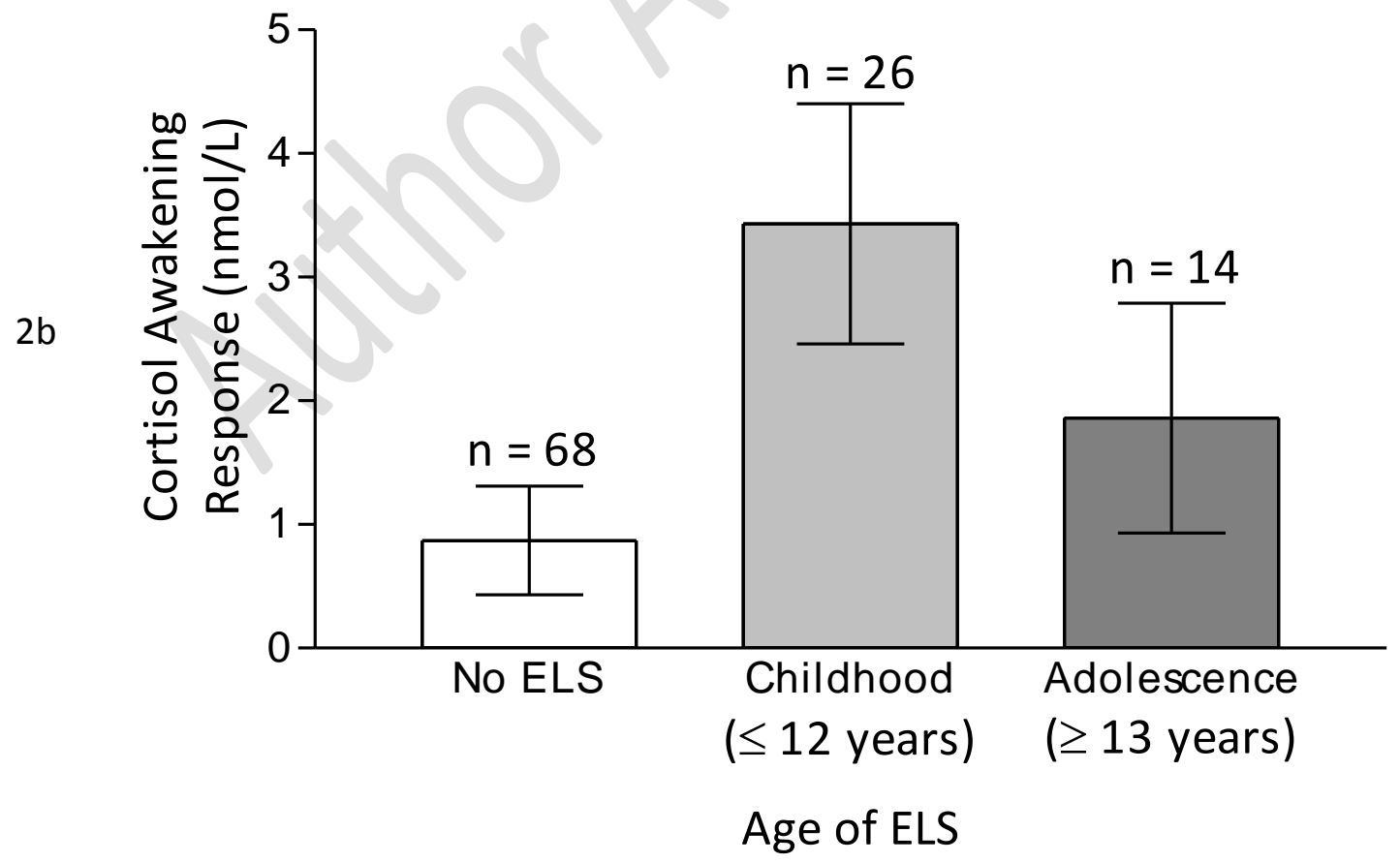


Figure 3: Scatter plot for the bivariate correlation between problems solved on first choice in the One Touch Stockings of Cambridge task and the cortisol awakening response $(\mathrm{p}=0.027, \mathrm{r}=-0.21, \mathrm{n}=$ 109).

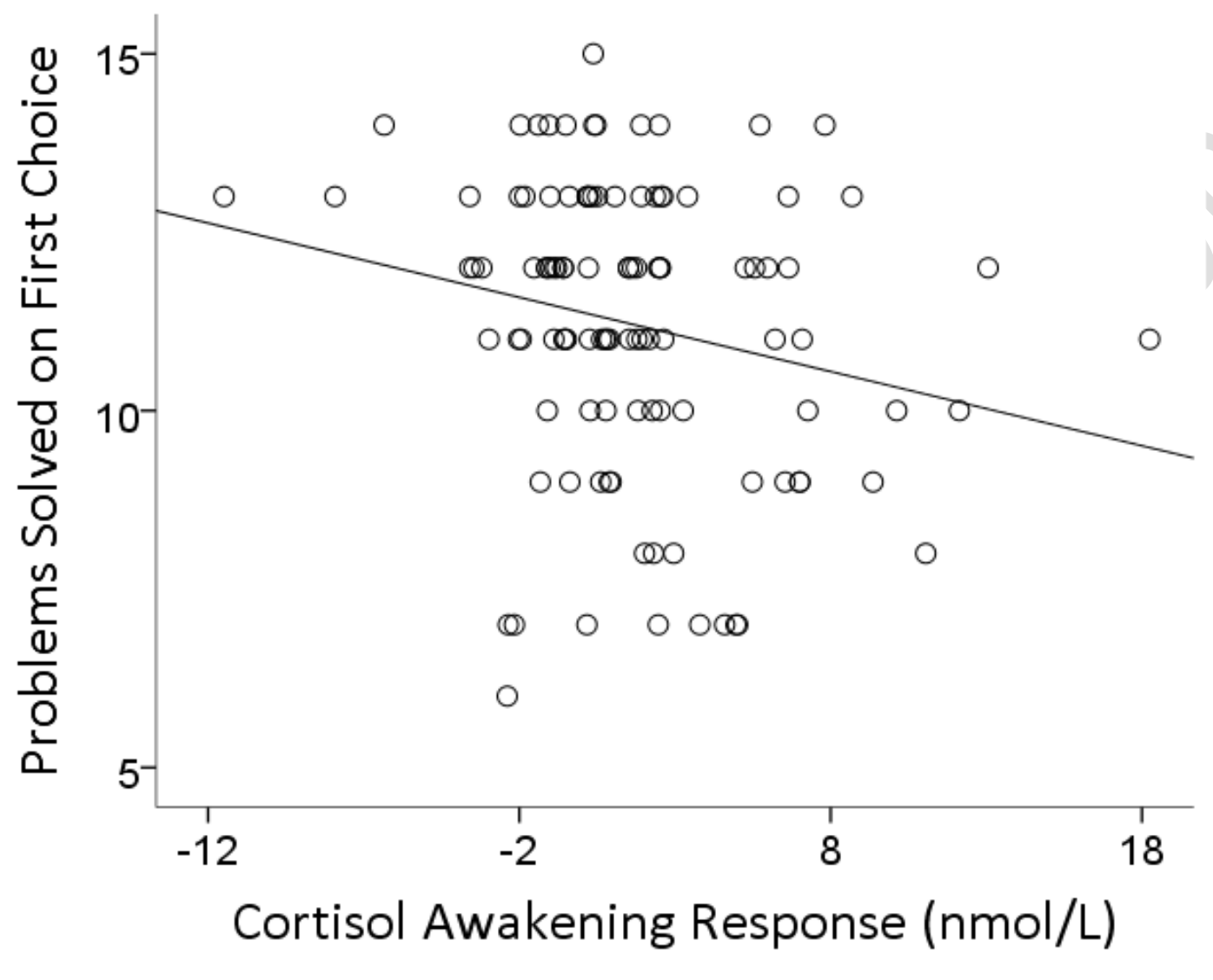

